\title{
UNCERTAINTY AVERSION AND EQUILIBRIUM EXISTENCE IN GAMES WITH INCOMPLETE INFORMATION
}

\author{
YARON AZRIELI AND ROEE TEPER ${ }^{\dagger}$
}

September 4, 2008

\begin{abstract}
We consider games with incomplete information à la Harsanyi, where the payoff of a player depends on an unknown state of nature as well as on the profile of chosen actions. As opposed to the standard model, the players in the game are not necessarily expected utility maximizers. Rather, their preferences over state-contingent utility vectors are represented by arbitrary functionals. Our first contribution is to provide simple and applicable definitions of both ex-ante and interim equilibria in this generalized setting. Second, we characterize equilibrium existence in terms of the preferences of the participating players. It turns out that, given some standard properties of the functionals, equilibrium exists in every game if and only if all players are averse to uncertainty. Finally, for a sub-class of preferences' representing functionals we show that there exists a symmetric equilibrium in every symmetric game if and only if all players share preferences.
\end{abstract}

Keywords: Games with incomplete information, Equilibrium existence, Uncertainty aversion.

JEL Classification: D81, C72.

\footnotetext{
${ }^{\dagger}$ School of Mathematical Sciences, Tel Aviv University, Tel Aviv 69978, Israel.

e-mail: yazrieli@gmail.com, teperroe@post.tau.ac.il

We thank Ehud Lehrer for many inspiring conversations. Also, we are grateful to Sophie Bade, Eran Hanany, Yuval Heller, David Schmeidler and Eilon Solan for helpful comments.
} 


\section{INTRODUCTION}

Since Harsanyi [21], games with incomplete information have proved to be a powerful tool in the analysis of strategic situations where agents are uncertain regarding the specifics of the environment. In the vast majority of applications, economists assume that the players in the game share a common prior probability distribution over the state space, and that each player is a Bayesian expected utility maximizer with respect to (w.r.t.) this prior (and given the strategies of his opponents). With these assumptions on players' preferences, the appropriate solution concept for such games is the Bayes-Nash equilibrium, either in its ex-ante or interim forms. Existence of Bayes-Nash equilibrium in every game with incomplete information is guaranteed by a fixed-point argument ${ }^{1}$.

Starting with Ellsberg [11], a rich literature has developed showing consistent violations of the expected utility maximization theory when decision makers are uncertain regarding the probabilities of relevant events. In particular, agents' preferences tend to exhibit uncertainty aversion, which cannot be explained within the subjective expected utility framework. The experimental findings of Ellsberg [11] and his successors inspired economists to develop alternative theories of decision making under uncertainty (e.g., Schmeidler [38], Gilboa and Schmeidler [18], and more recently Maccheroni et al. [30] and Lehrer [27]).

Roughly speaking, we interpret uncertainty as the situation where the probabilities of some relevant events are ambiguous, and cannot be determined by the decision maker ${ }^{2}$. Under this interpretation, uncertainty is present in many real-life game-like situations. For instance, a firm in a Cournot oligopoly may be too uncertain regarding the demand function to assign a probability to the event that the intercept of this function is between the numbers $\underline{a}$ and $\bar{a}$; An oil company bidding for the rights to drill in a new site may not have enough information to assess the probability that the site has a capacity of ten million barrels. Notice that the uncertainty in both these examples concerns the probabilities of payoff relevant states of nature, and not the strategies of the opponents. The firm in the first example may know the production levels of its competitors, and the oil company may know the bids of the other participants in the auction.

Given such examples, it is quite surprising that the literature remained relatively silent regarding uncertainty averse players in games with incomplete information. We should

\footnotetext{
${ }^{1}$ See Milgrom and Weber [31] for a general equilibrium existence result in games with incomplete information.

${ }^{2}$ As opposed to risk where the probabilities of outcomes are known. See Epstein [12].
} 
mention, however, several exceptions. Epstein and Wang [14] generalize the construction of a universal type space to a class of preferences that can accommodate uncertainty aversion. Kajii and Ui [22] introduce an equilibrium notion for games with incomplete information where players have maxmin preferences (Gilboa and Schmeidler [18]). Salo and Weber [35], Lo [29] and Bose et al. [7] study auctions with uncertainty averse bidders. Bade [5] shows existence of equilibrium in a political game where parties are uncertain regarding the distribution of voters.

The contribution of the current paper is twofold. First, we propose simple and applicable definitions of both ex-ante and interim equilibria in games with incomplete information when players' preferences over state-contingent utility vectors are represented by arbitrary functionals. We use the standard model of a game with incomplete information, where each one of a finite set of players is endowed with a partition of the state space ${ }^{3}$ that represents his information. Players' payoffs depend on the chosen action profile as well as on the realized state of nature. Thus, given the actions of other players, a player faces a state-dependent utility decision problem (see Karni [25] for a survey).

A strategy of a player is a function from states to (possibly mixed) actions that is measurable w.r.t. his partition. In any given state of nature, every strategy profile induces a probability distribution over pure action profiles. The utility of a player in this state of nature is his expected payoff according to this distribution. Thus, any strategy profile induces a real-valued function on the state space for each one of the players. We refer to such a function as the induced utility-vector.

In the ex-ante version of our equilibrium concept, each player $i$ is characterized by a functional $J_{i}$ over the space of real-valued functions over the state space. If $f$ is such a function then $J_{i}(f)$ represents the total utility that player $i$ derives from $f .{ }^{4}$ An ex-ante equilibrium is then naturally defined as a strategy profile such that no player $i$ can derive a higher utility (as measured by $J_{i}(f)$, where $f$ is the induced vector for player $i$ ) by altering his strategy. Notice that, as in standard Nash equilibrium, players beliefs about their opponents' strategies coincide with the $\operatorname{truth}^{5}$. In order to define interim equilibrium

\footnotetext{
${ }^{3}$ For simplicity, we restrict attention to finite state spaces. Our results can be extended to infinite spaces at the cost of adding standard technical assumptions on the various mathematical objects.

${ }^{4}$ Note that in the original Harsanyi model, $J_{i}(f)$ is the expected value of $f$ w.r.t. the common prior.

${ }^{5}$ As opposed to uncertainty averse players in normal-form games (see Mukerji and Tallon [32] for a survey), in our model players' uncertainty regarding their opponents' strategies is derived only from their uncertainty about the state of nature. An exception is the recent paper by Bade [4], where she
} 
we assume that each player $i$ is characterized by a family of functionals $\left\{J_{i}^{F}\right\}$, one for every non-empty event $F$. A strategy profile constitutes an interim equilibrium if, for every player $i$ and for every element $F$ in $i$ 's partition, the restriction to $F$ of the induced vector for $i$ maximizes player $i$ 's utility (as measured by $J_{i}^{F}$ ) given the strategies of $i$ 's opponents.

To illustrate the definition of equilibrium, as well as to motivate the main result of the paper, consider the following example of a game with incomplete information. There are two players $i=1,2$ and two states $\left\{s_{1}, s_{2}\right\}$. The action set of player 1 is $\{T, B\}$ and that of player 2 is $\{L, R\}$. The information partitions of both players are trivial. The payoffs are described in the diagram below, where 1 chooses row and 2 column.
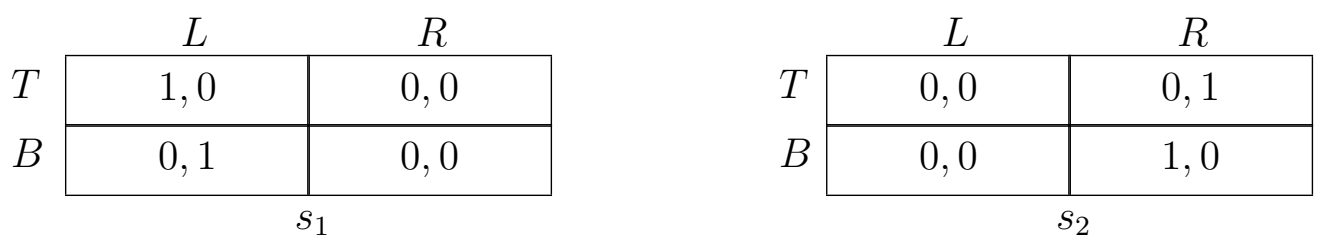

Consider the case where both players are 'optimistic' in the sense that they evaluate any utility vector according to its maximal element. That is, if $f=\left(f\left(s_{1}\right), f\left(s_{2}\right)\right)$ is the induced state-contingent utility for player $i$ when a certain strategy profile is played, then $i$ 's total utility is $J_{i}(f)=\max \left\{f\left(s_{1}\right), f\left(s_{2}\right)\right\}(i=1,2)$.

We claim that with these preferences of the players there is no equilibrium in this game (since both players have trivial information partitions the ex-ante and interim versions coincide). Indeed, assume that player 1 plays $\beta T+(1-\beta) B$, and 2 plays $\alpha L+(1-\alpha) R$ for some $0 \leq \alpha, \beta \leq 1$. Then the induced vector for player 1 is $(\alpha \beta,(1-\alpha)(1-\beta))$ and for player 2 is $((1-\beta) \alpha, \beta(1-\alpha))$. Therefore, the images of the best response correspondences of both players consist of only pure strategies. But it is easy to see that there is no pure equilibrium in this game and, therefore, no equilibrium exists.

As the above example shows, some restrictions must be imposed on the preferences of the participating players in order for equilibrium to exist. We are therefore led to ask what characterizes preferences that guarantee equilibrium existence in every game. Our second and main contribution is to show that, given some natural and standard properties of the functionals $J_{i}$, equilibrium exists in every game if and only if all the players

considers incomplete information extensions à la Aumann [2] of normal-form games. However, her results are confined to the case where payoffs are state-independent. 
are (weakly) uncertainty averse. By uncertainty aversion we mean concavity of the preferences representing functional ${ }^{6}$. The properties that we assume each of the functionals possesses are either continuity and translation invariance (Theorem 1) or continuity, homogeneity, monotonicity and non-degeneracy (Theorem 2). These theorems (as well as the other results of the paper) are stated for the ex-ante version of the equilibrium concept, but similar results can be phrased for the interim version as well. We choose to present the results for the ex-ante version of the equilibrium since it simplifies notation and proofs.

We believe that this result is of interest to economists for three reasons. First and foremost, finding necessary and sufficient conditions for existence is the most basic task for any solution concept. By so doing, the boundaries of the domain where the concept can be applied are clearly marked. Second, since agents' preferences often exhibit uncertainty aversion, our result implies that in many cases equilibrium existence is guaranteed $^{7}$. Third, the result provides a possible explanation for why in some game-like situations stability is not achieved.

We proceed as follows. Section 2 formally defines the class of games with incomplete information that we consider and the notion of ex-ante equilibrium. In Section 3 we present several properties of the preferences representing functionals and state the main results which relate uncertainty aversion to equilibrium existence. In the next Section 4 we discuss in more detail some of the more familiar functional forms that are often used in the literature. Namely, we study the implications of our result to maxmin preferences (Gilboa and Schmeidler [18]), variational preferences (Maccheroni et al. [30]), Choquet preferences (Schmeidler [38]) and concave integral preferences (Lehrer [26]).

A natural question in our setting is what characterizes the case where different players share the same preferences. We give an answer to this question in Section 5 for the cases of maxmin and concave integral preferences. The characterization is based on the notions of symmetric games and symmetric equilibrium. To the best of our knowledge, this characterization is new even in the special case of expected utility preferences. In Section 6 we define interim equilibrium and briefly discuss the subject of updating of preferences and dynamic consistency in our multi-agent environment. Remarks are in Section 7, and finally, all the proofs are in Section 8.

\footnotetext{
${ }^{6}$ For a further discussion see Section 3.

${ }^{7}$ It should be noted however that even if only one of the agents is not uncertainty averse then it is possible that the set of equilibria is empty.
} 


\section{EX-ANTE EQUILIBRIUM}

An environment is a tuple $\left(S, N, \mathfrak{J}=\left\{J_{i}\right\}_{i \in N}\right)$. The first component $S=\left\{s_{1}, s_{2}, \ldots, s_{m}\right\}$ is a non-empty finite set of states of nature (the state space). We assume $m \geq 2$ throughout. A utility-vector (vector, for short) is any function from $S$ to $\mathbb{R}$. We will usually use the letters $f, g$ to denote vectors. Addition of vectors and multiplication of vectors by scalars are performed pointwise. We can therefore identify the space of all vectors with the linear space $\mathbb{R}^{m}$. The constant vector $f$ in which $f(s)=c$ for every $s \in S$ will be denoted by $\underline{c}$. $N=\{1,2, \ldots, n\}$ where $n \geq 2$ is the set of players. For each $i \in N$, the functional $J_{i}: \mathbb{R}^{m} \rightarrow \mathbb{R}$ represents player $i$ 's preferences over vectors ${ }^{8}$. We fix the environment until Section 6.

A normal-form game with incomplete information (game, for short) $G$ is defined by $G=\left(\left\{F_{i}\right\}_{i \in N},\left\{A_{i}\right\}_{i \in N},\left\{u_{i}\right\}_{i \in N}\right)$. For each $i \in N, F_{i}$ is player $i$ 's information partition a partition of $S$, and $A_{i}$ is the finite non-empty set of actions of $i .{ }^{9}$ Denote by $A=\times_{i \in N} A_{i}$ the set of all action profiles with typical element $a=\left(a_{1}, \ldots, a_{n}\right)$. The utility function of player $i \in N$ is $u_{i}: S \times A \rightarrow \mathbb{R}$. The set of all games (in a fixed environment) is denoted by $\Gamma$.

Let $G \in \Gamma$ and fix $i \in N$. A strategy for player $i$ is an $F_{i}$-measurable function ${ }^{10}$ $\sigma_{i}: S \rightarrow \Delta\left(A_{i}\right)$. The set of all strategies for player $i$ is denoted $\Sigma_{i}$, and the set of all strategy profiles is $\Sigma=\times_{i \in N} \Sigma_{i}$ with typical element $\sigma=\left(\sigma_{1}, \ldots, \sigma_{n}\right)$. The probability with which player $i$ plays the action $a_{i} \in A_{i}$ in state $s \in S$ according to $\sigma_{i}$ is denoted $\sigma_{i}\left(s, a_{i}\right)$. As usual, $\sigma_{-i}$ denotes the strategy profile of players other than $i$ in which each player $j \neq i$ plays as in $\sigma$.

Every strategy profile $\sigma$ in a game $G$ induces a vector for each one of the players. Formally, the induced vector of player $i$ is $f_{i}^{(\sigma)}$ where $f_{i}^{(\sigma)}(s)=\sum_{a \in A}\left(\prod_{j \in N} \sigma_{j}\left(s, a_{j}\right)\right) u_{i}(s, a)$ for every $s \in S$. We can now define our notion of ex-ante equilibrium.

\footnotetext{
${ }^{8}$ Usually, the preferences of agents are given by a binary relation. Our interest here is in the functional properties that guarantee equilibrium existence. Therefore, we assume all that is needed for preferences to be represented by functionals. See Bade [4] for a notion of equilibrium where preferences are given by a binary relation.

${ }^{9}$ Here we assume that each player has the same set of actions in all states of nature. Our results can be easily extended to the case where the action set of a player vary across $S$ as long as it is constant on each element of that player's information partition.

${ }^{10}$ For a finite set $X, \Delta(X)$ is the set of all probability measures over $X$.
} 
Definition 1. Let $G \in \Gamma$. A strategy profile $\sigma \in \Sigma$ is an ex-ante $\mathfrak{J}$-equilibrium of $G$ if, for every $i \in N$ and for every $\sigma_{i}^{\prime} \in \Sigma_{i}, J_{i}\left(f_{i}^{(\sigma)}\right) \geq J_{i}\left(f_{i}^{\left(\sigma_{i}^{\prime}, \sigma_{-i}\right)}\right)$.

\section{UNCERTAINTY AVERSION AND EX-ANTE EQUILIBRIUM EXISTENCE}

The following axioms are standard in the theory of decision making, and are usually stated as properties of preference orders. Since we assume that preferences are already represented by functionals, we consider the analogous functional properties. In each one of the following axioms we mean that the relevant property holds for every $J_{i} \in \mathfrak{J}$.

Continuity (C): $J_{i}$ is continuous over $\mathbb{R}^{m}$.

Homogeneity $\mathbf{( H )}$ : For every vector $f$ and for every $\alpha \geq 0, J_{i}(\alpha f)=\alpha J_{i}(f)$.

Monotonicity (M): For every two vectors $f, g$, if $f(s) \geq g(s)$ for every $s \in S$ then $J_{i}(f) \geq J_{i}(g)$.

Translation Invariance (TI): For every vector $f$ and a constant vector $\underline{c}, J_{i}(f+\underline{c})=$ $J_{i}(f)+c$.

Non-degeneracy (ND): There exist vectors $f, g$ such that $J_{i}(f)>J_{i}(g)$.

Uncertainty Aversion (UA): $J_{i}$ is concave. That is, $J_{i}(\alpha f+(1-\alpha) g) \geq \alpha J_{i}(f)+$ $(1-\alpha) J_{i}(g)$ for every two vectors $f, g$ and $\alpha \in(0,1)$.

Schmeidler's [38] seminal definition of uncertainty aversion ${ }^{11}$ states that if the acts $f, g$ satisfy $f \succeq g$, then for any $\alpha \in(0,1), \alpha f+(1-\alpha) g \succeq g$. This axiom translates into quasiconcavity of the preferences representing functional (see Cerreia et al. [8] and Hanany and Klibanoff [20]). However, to quote Schmeidler, "Intuitively, uncertainty aversion means that "smoothing" or averaging utility distributions makes the decision maker better off ... Concavity captures best the heuristic meaning of uncertainty aversion". We note that our main interest is in proving that ex-ante equilibrium existence implies aversion to uncertainty by all agents. Thus, adapting a stronger version of the (UA) axiom gives a stronger result.

While the former axioms are relevant to the preferences of each decision maker separately, the next axiom reflects the interactive flavor of our model. Namely, the last axiom states that ex-ante $\mathfrak{J}$-equilibrium exists in every game $G \in \Gamma$. It should be emphasized

\footnotetext{
${ }^{11}$ While this is the most commonly used definition of uncertainty aversion, there are alternative definitions in the literature such as those of Epstein [12] and Ghirardato and Marinacci [17].
} 
that we do not think that equilibrium existence is a plausible property of players' preferences neither from the descriptive nor from the normative points of view. We state this property as an axiom just for convenience of use in the sequel.

Equilibrium Existence (EE): There exists an ex-ante $\mathfrak{J}$-equilibrium in every game $G \in \Gamma$.

We can now state our main results.

Theorem 1. If $(C)$ and $(T I)$ are satisfied then $(U A)$ is equivalent to $(E E)$.

Remark 1. In order to guarantee ex-ante equilibrium existence in every game it is sufficient that each of the functionals $J_{i}$ be quasi-concave and continuous. Thus, $(U A)$ implies (EE) without any further assumption.

Remark 2. Since the combination of $(M)$ and $(T I)$ implies $(C)$, Theorem 1 remains valid if $(C)$ is replaced by $(M)$.

Our next aim is to obtain a similar result to that of Theorem 1 when (TI) is replaced by $(\mathrm{H})$. Notice that Theorem 1 and Remark 1 imply that, given (C) and (TI), the seemingly stronger property of concavity is equivalent to quasi-concavity. The fact that a continuous, monotone and homogeneous functional on ${ }^{12} \mathbb{R}_{+}^{m}$ is concave iff it is quasiconcave is already known (see Rader [33], page 98 Theorem 6, and Shephard [41], page 31 Proposition 7). In the next theorem we restrict attention to the class of games $G \in \Gamma$ with non-negative payoffs and, correspondingly, to functionals with $\mathbb{R}_{+}^{m}$ as a domain.

Theorem 2. If $(C),(H),(M)$ and $(N D)$ are satisfied then $(U A)$ is equivalent to $(E E)$.

Remark 3. Theorem 2 is not true without the restriction of the domain to the nonnegative orthant. Indeed, let $m=2$ and $J_{i}(f)=\max \left\{f\left(s_{1}\right)+f\left(s_{2}\right), 2\left(f\left(s_{1}\right)+f\left(s_{2}\right)\right)\right\}$ for every $i \in N$. Then each $J_{i}$ is homogenous, (strictly) monotone, continuous, quasiconcave but not concave. Thus, $(E E)$ is satisfied while $(U A)$ is not.

\section{Special FUnCtional FORMS}

4.1. Maxmin preferences. Gilboa and Schmeidler [18] axiomatize preference orders that are determined by the minimal expected utility w.r.t. some convex and compact

${ }^{12} \mathbb{R}_{+}^{m}$ is the set of all non-negative utility vectors. 
set of priors. Given a set of probability measures $P_{i} \subseteq \Delta(S)$, we say that $J_{i}$ represents maxmin preferences w.r.t. $P_{i}$ if $^{13} J_{i}(f)=\min _{p \in P_{i}} p \cdot f$ for every vector $f$.

The following is a consequence of the Gilboa-Schmeidler axiomatization (see in particular Lemma 3.5 in [18]), when combined with Theorem 1 and Remark 2 above. It can be seen as an alternative characterization of maxmin preferences.

Corollary 1. The functionals $\left\{J_{i}\right\}_{i \in N}$ satisfy $(H),(M),(T I)$ and $(E E)$ if and only if there is a family of sets $\left\{P_{i}\right\}_{i \in N}$, where $P_{i} \subseteq \Delta(S)$ is convex and compact for each $i$, such that $J_{i}$ represents maxmin preferences w.r.t. $P_{i}$. Moreover, each set $P_{i}$ is uniquely determined by $J_{i}$.

Maccheroni et al. [30] axiomatize variational preferences, which generalize the model of maxmin preferences. The functional form of a variational preference is $J_{i}(f)=$ $\min _{p \in \Delta(S)}(p \cdot f+c(p))$ for every vector $f$, where $c: \Delta(S) \rightarrow[0, \infty]$ is a grounded ${ }^{14}$ convex and lower-semicontinuous functional. A consequence of the axiomatization of Maccheroni et al. along with Theorem 1 and Remark 2 is the following.

Corollary 2. The functionals $\left\{J_{i}\right\}_{i \in N}$ satisfy $(M),(T I)$ and $(E E)$ if and only if every $J_{i}$ represents a variational preference.

Remark 4. Similar functional forms to the maxmin and variational preferences appear in the risk assessment literature (see Artzner et al. [1], Delbaen [9], and Föllmer and Schied [16]).

4.2. Non-additive integration. Choquet integral preferences (Schmeidler [38]) are often used as an alternative to expected utility maximization. It is well known that the Choquet integral w.r.t. any capacity ${ }^{15} v$ satisfies the $(M),(H)$ and $(T I)$ properties. If $v$ is convex $(v(E)+v(F) \leq v(E \cup F)+v(E \cap F)$ for every two events $E, F \subseteq S)$ then the Choquet integral w.r.t. $v$ is a concave functional. Moreover, it coincides with the maxmin functional w.r.t. the core ${ }^{16}$ of $v$.

\footnotetext{
${ }^{13}$ If $p \in \Delta(S)$ and $f \in \mathbb{R}^{m}$ is a vector then $p \cdot f=\int_{S} f d p$ denotes the expected value of $f$ according to the probability measure $p$.

${ }^{14} \mathrm{~A}$ function $c$ is grounded if its infimum over the domain is zero.

${ }^{15} \mathrm{~A}$ capacity is a set function $v: 2^{S} \rightarrow \mathbb{R}$ satisfying $v(\emptyset)=0, v(S)=1$ and $v(E) \leq v(F)$ whenever $E \subseteq F$.

${ }^{16}$ The core of a capacity $v$ is the set of probability measures $p$ over $S$ satisfying $p(E) \geq v(E)$ for every event $E$.
} 
However, if $v$ is not convex then the Choquet integral w.r.t. $v$ is not a concave functional. In this case, Theorem 1 implies that, if one of the players has Choquet preferences w.r.t. a non-convex capacity, then there is a game $G$ with no ex-ante $\mathfrak{J}$-equilibrium. Note that in the example presented in the introduction both players' preferences are represented by the Choquet integral w.r.t. the capacity that assigns 1 to every non-empty set of states. This capacity is non-convex, and indeed, the proposed game has no equilibrium. This example can be easily generalized to provide a constructive proof of ex-ante equilibrium non-existence whenever one of the players has Choquet preferences w.r.t. some non-convex capacity.

Recently, Lehrer [26] introduced the concave integral for capacities. This integral is defined only for non-negative vectors. Therefore, in the rest of this subsection we restrict attention to the orthant of non-negative vectors and, correspondingly, to games with non-negative payoffs. Given a non-negative vector $f$, the concave integral of $f$ w.r.t. a capacity $v$ is defined by

$$
\int f d v:=\max \left\{\sum_{A \subseteq S} \lambda_{A} v(A) ; \sum_{A \subseteq S} \lambda_{A} \mathbb{1}_{A} \leq f, \lambda_{A} \geq 0\right\},
$$

where $\mathbb{1}_{A}$ is the indicator function of $A$. Notice that if $v$ is additive then the righthand side of (1) is the Lebesgue integral of $f$.

As implied by its name, the concave integral w.r.t. any capacity $v$ is a concave functional over the set of non-negative vectors. Lehrer [26] shows that it is continuous even on the boundary of its domain. By Remark 1 we have:

Corollary 3. If, for every $i \in N$, the functional $J_{i}$ is defined by the concave integral w.r.t. some capacity $v_{i}$ then $(E E)$ is satisfied.

Note that the concave integral is also monotone and homogeneous (and trivially nondegenerate). Thus, the concave integral satisfies the properties in Theorem 2, however it is not characterized by these properties (Lehrer [26]).

Unlike the Choquet integral, $(T I)$ is not always satisfied. A capacity $v$ has a large core (Sharkey [40]) if for every additive measure ${ }^{17} \mu \geq v$ there exists a core element $p$ of $v$ such that $\mu \geq p$. Azrieli and Lehrer [3] proved that the concave integral w.r.t. $v$ satisfies $(T I)$ if and only if $v$ has a large core. In this case the concave integral coincides with the maxmin functional, where the convex compact set of priors is the core of $v$.

\footnotetext{
${ }^{17}$ Not necessarily a probability measure.
} 


\section{Symmetry AND COMMON PREFERENCES}

A natural question in our multi-agent environment is what characterizes the case where different players have the same preferences. We restrict attention to the special cases of maxmin preferences and of preferences that can be represented by the concave integral. As was shown in the previous section, in both these cases ex-ante equilibrium exits in every game. The characterization is based on the notions of symmetric games and symmetric ex-ante equilibrium, which we now define.

Fix two players $i, j \in N$. For a given action profile $a$ in some game $G \in \Gamma$ with $A_{i}=A_{j}$, we denote $a^{i j}$ the action profile in which players $i$ and $j$ exchange their actions while any other player plays the same as in $a$. That is, $a_{i}^{i j}=a_{j}, a_{j}^{i j}=a_{i}$ and $a_{k}^{i j}=a_{k}$ for every $k \in N \backslash\{i, j\}$.

Definition 2. A game $G \in \Gamma$ is $i j$-symmetric if $F_{i}=F_{j}, A_{i}=A_{j}$, and for every $s \in S$ and $a \in A, u_{i}(s, a)=u_{j}\left(s, a^{i j}\right)$ and $u_{k}(s, a)=u_{k}\left(s, a^{i j}\right)$ for every player $k \neq i, j$.

We now state an additional axiom and then use it to characterize the case of common preferences.

$\boldsymbol{i j}$-Symmetric Equilibrium ( $\boldsymbol{i j}$-SE): If $G$ is $i j$-symmetric then there is an ex-ante $\mathfrak{J}$-equilibrium $\sigma$ in $G$ such that $\sigma_{i}=\sigma_{j}$.

\subsection{Maxmin and common multiple--priors.}

Proposition 1. If, for some two players $i, j \in N$, the functionals $J_{i}$ and $J_{j}$ represent maxmin preferences w.r.t. $P_{i}$ and $P_{j}$ respectively, then (ij-SE) is satisfied if and only if $P_{i}=P_{j}$

A game $G \in \Gamma$ is symmetric if it is $i j$-symmetric for every two players $i, j$. A symmetric ex-ante $\mathfrak{J}$-equilibrium (in a symmetric game $G$ ) is an ex-ante $\mathfrak{J}$-equilibrium $\sigma$ such that $\sigma_{i}=\sigma_{j}$ for every $i, j \in N$. By a simple adjustment of the proof of Proposition 1 one obtains the following corollary.

Corollary 4. If, for every $i \in N$, the functional $J_{i}$ represents maxmin preferences w.r.t. $P_{i}$, then there is a symmetric ex-ante $\mathfrak{J}$-equilibrium in every symmetric game if and only if all the $P_{i}$ 's are equal. 
5.2. The concave integral and common capacities. Let $v_{i}$ be the uniform probability distribution over $\{1,2\}$, and $v_{j}$ be the capacity defined by $v_{j}(A)=1$ for every non-empty subset $A$ of $\{1,2\}$. Even though these capacities are different, if $J_{i}$ and $J_{j}$ are defined by the concave integral w.r.t. $v_{i}$ and $v_{j}$ accordingly, then $J_{i}$ and $J_{j}$ represent the same preference order.

The normalized cover (cover, for short) of a capacity $v$ is defined by

$$
\hat{v}(A)=\frac{\int \mathbb{1}_{A} d v}{\int \underline{1} d v}
$$

for every $A \subseteq S$.

Proposition 2. If, for some two players $i, j \in N$, the functionals $J_{i}$ and $J_{j}$ are defined by the concave integral w.r.t. $v_{i}$ and $v_{j}$ respectively, then (ij-SE) is satisfied if and only if $\hat{v}_{i}=\hat{v}_{j}$.

Corollary 5. If, for every $i \in N$, the functional $J_{i}$ is defined by the concave integral w.r.t. $v_{i}$, then there is a symmetric ex-ante $\mathfrak{J}$-equilibrium in every symmetric game if and only if all the $\hat{v}_{i}$ 's are equal.

\section{INTERIM EQUILIBRIUM}

This section is devoted to the notion of interim equilibrium and its existence. However, in order to define interim equilibrium we first need to revise the notion of an environment. An interim environment is a tuple $\left(S, N, \mathfrak{J}=\left\{J_{i}^{F}\right\}_{i \in N, F \subseteq S}\right)$. The first two components $S$ and $N$ are as described in Section 2. Given an event $F \subseteq S$, an $F$-vector is any function from $F$ to $\mathbb{R}$. Once $F$ is fixed we identify the space of all $F$-vectors with the linear space $\mathbb{R}^{|F|}$. For each $i \in N$ and $F \subseteq S$, the functional $J_{i}^{F}: \mathbb{R}^{|F|} \rightarrow \mathbb{R}$ represents player $i$ 's preferences over $F$-vectors.

Given such an environment, a game with incomplete information, a strategy, a strategy profile and an induced vector (over S) are defined as in Section 2. Given a vector $f$ over $S$ and an event $F \subseteq S$, we denote by $\left.f\right|_{F}$ the $F$-vector which is the restriction of $f$ to $F$.

Definition 3. Let $G \in \Gamma$. A strategy profile $\sigma \in \Sigma$ is an interim $\mathfrak{J}$-equilibrium of $G$ if, for every $i \in N$, every $F \in F_{i}$, and for every $\sigma_{i}^{\prime} \in \Sigma_{i}, J_{i}^{F}\left(\left.f_{i}^{(\sigma)}\right|_{F}\right) \geq J_{i}^{F}\left(\left.f_{i}^{\left(\sigma_{i}^{\prime}, \sigma_{-i}\right)}\right|_{F}\right)$.

Note that when considering the notion of interim environment, the analogous definition of an ex-ante $\mathfrak{J}$-equilibrium in a game $G$ is a strategy profile $\sigma$, such that for every 
$i \in N$ and for every $\sigma_{i}^{\prime} \in \Sigma_{i}, J_{i}^{S}\left(f_{i}^{(\sigma)}\right) \geq J_{i}^{S}\left(f_{i}^{\left(\sigma_{i}^{\prime}, \sigma_{-i}\right)}\right)$. In this revised model, the axioms presented in Section 3 can be reformulated as possible properties of $J_{i}^{S}$ (instead of $J_{i}$ ), resulting with the analogous to Theorems 1 and 2 .

In order to characterize interim $\mathfrak{J}$-equilibrium existence, we need to adapt the axioms presented in Section 3 to suit the interim environment. By "adapt" we mean that each relevant property (depending on the axiom) holds for every $J_{i}^{F}$. Now, Theorems 1 and 2 can be reformulated to show that interim $\mathfrak{J}$-equilibrium exists in every game if and only if all players are averse to uncertainty.

In the Harsanyi [21] model, ex-ante and interim preferences are related through Bayes rule. A well-known result in this case is that the sets of ex-ante and interim equilibria are equivalent. This raises two tangled questions regarding the interim environment presented above.

The first question concerns the relation between $J_{i}^{S}$ and $J_{i}^{F}$. Informally, what is (or should be) the relation between player $i$ 's preferences before and after receiving the information that an event $F$ occurred? The matter of updating preferences has been extensively dealt with in the literature. Examples include updating rules for Choquet preferences (e.g., Dempster [10] and Shafer [39]), and full Bayesian (e.g., Sarin and Wakker [37] and Fagin and Halpern [15]) and maximum-likelihood Bayesian (Gilboa and Schmeidler [19]) updating rules for Maxmin preferences. Updating general preferences, not necessarily any of the mentioned above, were studied in Hanany and Klibanof [20] and Wang $[42]^{18}$.

An important property of an updating rule is dynamic consistency. To quote Epstein and Le Breton ([13], page 3), “...the assumption of dynamic consistency is clearly advantageous in terms of theoretical elegance and simplicity and also analytical tractability, and so is "natural" for these reasons. From a normative point of view, it is difficult to imagine adopting or recommending a dynamically inconsistent updating rule for use of statistical decision problem." Epstein and Le Breton show that if preferences that are "based on beliefs" admit dynamic consistent updating, then Bayesianism is implied. This leads us to ask the second question. Applying which updating rules, players will be dynamic consistent and the two equilibria concepts presented will be equivalent? These questions are beyond the scope of this paper and are left for future work.

\footnotetext{
${ }^{18}$ These are only partial lists out of the vast literature dealing with updating preferences.
} 


\section{FinAl COMMENTS}

7.1. General common preferences. In Section 5 we characterize, for maxmin and concave integral preferences, the case players' preferences representing functionals are the same. An interesting question is whether these results can be stated for general functionals as in Theorems 1 and 2. We formulate this in the following two conjectures.

Conjecture 1. Assume that every $J_{i}$ satisfies (C),(TI) and (EE). Then in every symmetric game there exists a symmetric ex-ante $\mathfrak{J}$-equilibrium if and only if all the $J_{i}$ 's are equal.

Conjecture 2. Assume that, for every $i \in N, J_{i}$ is defined over $\mathbb{R}_{+}^{m}, J_{i}(\underline{1})=1$, and satisfies $(C),(H),(M),(N D)$ and (EE). Then in every symmetric game with non-negative payoffs there exists a symmetric ex-ante $\mathfrak{J}$-equilibrium if and only if all the $J_{i}$ 's are equal.

The normalization requirement in Conjecture 2 (which is satisfied by $(T I)$ in Conjecture 1) is due to the fact that, if all $J_{i}$ 's represent the same preferences, then there exists a symmetric ex-ante $\mathfrak{J}$-equilibrium in every symmetric game. Additionally, the following example shows that if the $J_{i}$ 's are defined over the entire space, then Conjecture 2 does not hold. Indeed, the functionals $J_{1}\left(f_{1}, f_{2}\right)=\frac{1}{2}\left(f_{1}+f_{2}\right)$ and $J_{2}\left(f_{1}, f_{2}\right)=$ $\min \left\{\frac{1}{2}\left(f_{1}+f_{2}\right), f_{1}+f_{2}\right\}$ defined over $\mathbb{R}^{2}$ satisfy all conditions of Conjecture 2 . However, they are different and represent the same preferences.

7.2. Zero-sum games and a 'grain of agreement'. Consider the result of Corollary 4. It characterizes the case where all the players have exactly the same set of priors which they think are possible. One may be interested in the weaker property that there is at least one prior which all the players think is possible. In other words, assuming that the each player $i$ in the environment has maxmin preferences w.r.t. the (convex compact) set $P_{i}$, when is the intersection $\bigcap_{i \in N} P_{i}$ non-void? ${ }^{19}$

The answer to the above question is given by the following proposition. A game $G \in \Gamma$ is called zero-sum if $\sum_{i \in N} u_{i}(a, s)=0$ for every $a \in A$ and $s \in S$.

Proposition 3. Assume that each $J_{i}$ represents maxmin preferences w.r.t. $P_{i}$. Then the following conditions are equivalent:

(i) $\bigcap_{i \in N} P_{i} \neq \emptyset$.

\footnotetext{
${ }^{19} \mathrm{~A}$ similar question was studied by Billot et al. [6], Kajii and Ui [24] and Rigotti et al. [34] in the setting of a exchange economy, and by Kajii and Ui [23] in the setting of bets between two agents.
} 
(ii) In every strategy profile $\sigma \in \Sigma$ of every zero-sum game $G$, if $J_{i}\left(f_{i}^{(\sigma)}\right)>0$ for some $i \in N$ then there is $j \in N$ such that $J_{j}\left(f_{j}^{(\sigma)}\right) \leq 0$.

\section{Proofs}

8.1. Proofs of Section 3. We start with the following lemma.

Lemma 1. Assume $J_{i}$ satisfies $(C)$. If $J_{i}$ is not concave then there are vectors $f, g$ such that $J_{i}(\alpha f+(1-\alpha) g)<\alpha J_{i}(f)+(1-\alpha) J_{i}(g)$ for every $\alpha \in(0,1)$.

Proof. If $J_{i}$ is not concave then there are vectors $f^{\prime}, g^{\prime}$ and $\alpha_{0} \in(0,1)$ such that $J_{i}\left(\alpha_{0} f^{\prime}+\right.$ $\left.\left(1-\alpha_{0}\right) g^{\prime}\right)<\alpha_{0} J_{i}\left(f^{\prime}\right)+\left(1-\alpha_{0}\right) J_{i}\left(g^{\prime}\right)$. By $(\mathrm{C})$, the set $B:=\left\{\beta \in[0,1]: J_{i}\left(\beta f^{\prime}+(1-\right.\right.$ $\left.\left.\beta) g^{\prime}\right) \leq \beta J_{i}\left(f^{\prime}\right)+(1-\beta) J_{i}\left(g^{\prime}\right)\right\}$ is compact and contains an interval around $\alpha_{0}$. Let $\beta_{1}$ be the minimal element of $B$ which is larger than $\alpha_{0}$ and satisfies $J_{i}\left(\beta_{1} f^{\prime}+\left(1-\beta_{1}\right) g^{\prime}\right)=$ $\beta_{1} J_{i}\left(f^{\prime}\right)+\left(1-\beta_{1}\right) J_{i}\left(g^{\prime}\right)$. Similarly, let $\beta_{2}$ be the maximal element of $B$ which is smaller than $\alpha_{0}$ and satisfies $J_{i}\left(\beta_{2} f^{\prime}+\left(1-\beta_{2}\right) g^{\prime}\right)=\beta_{2} J_{i}\left(f^{\prime}\right)+\left(1-\beta_{2}\right) J_{i}\left(g^{\prime}\right)$. Define $f=$ $\beta_{1} f^{\prime}+\left(1-\beta_{1}\right) g^{\prime}$ and $g=\beta_{2} f^{\prime}+\left(1-\beta_{2}\right) g^{\prime}$. Then $J_{i}(\alpha f+(1-\alpha) g)<\alpha J_{i}(f)+(1-\alpha) J_{i}(g)$ for every $\alpha \in(0,1)$.

\section{The proof of Theorem 1:}

$$
(\mathbf{E E}) \Longrightarrow(\mathbf{U A})
$$

Assume to the contrary that (C), (TI) and (EE) are satisfied, and that there is $i \in N$ such that $J_{i}$ is not concave. Consider the following game $G \in \Gamma$. The information partitions of all the players are trivial. The action set of player $i$ is $A_{i}=\{T, B\}$ and the action set of some arbitrary player $j \neq i$ is $A_{j}=\{L, R\}$. Each one of the other players (if there are any) has only one action and, therefore, these players have no influence on the outcome of the game.

The payoff function for player $j$ is given by

$$
u_{j}\left(s, a_{i}, a_{j}\right)= \begin{cases}1 & ; a_{i}=T, a_{j}=L \\ 1 & ; a_{i}=B, a_{j}=R \\ 0 & ; a_{i}=T, a_{j}=R \\ 0 & ; a_{i}=B, a_{j}=L .\end{cases}
$$

Let $f, g$ be as in Lemma 1. Choose a number $M>0$ large enough such that $J_{i}(f+\underline{M})>$ $J_{i}(g)$ and $J_{i}(g+\underline{M})>J_{i}(f)$. Existence of such a number is guaranteed by (TI). Let the 
payoff function to player $i$ be given by

$$
u_{i}\left(s, a_{i}, a_{j}\right)= \begin{cases}f(s) & ; a_{i}=T, a_{j}=L \\ g(s) & ; a_{i}=B, a_{j}=R \\ f(s)+M & ; a_{i}=T, a_{j}=R \\ g(s)+M & ; a_{i}=B, a_{j}=L .\end{cases}
$$

Thus, the resulting bimatrix game in state $s \in S$ is given by the following diagram, where $i$ is the rows player and $j$ the columns player.

$$
\begin{array}{l|c|c|}
\multicolumn{2}{c}{L} & \multicolumn{1}{c}{R} \\
\cline { 2 - 3 } T & f(s), 1 & f(s)+M, 0 \\
\cline { 2 - 3 } B & g(s)+M, 0 & g(s), 1 \\
\cline { 2 - 3 } & &
\end{array}
$$

We show that $G$ has no ex-ante $\mathfrak{J}$-equilibrium. First, it is easy to check that there is no pure ex-ante $\mathfrak{J}$-equilibrium in $G$ (recall that the information partitions are trivial so both players must play the same strategy in all states of nature).

Now, assume that player $j$ plays the strategy $\alpha L+(1-\alpha) R$ for some $\alpha \in[0,1]$. By (TI) and the construction of $f, g$ it cannot be that $f-g$ is constant since in this case $J_{i}$ is linear on the interval $[f, g]$. It follows that $\alpha f+(1-\alpha)(f+\underline{M}) \neq \alpha(g+\underline{M})+(1-\alpha) g$, so every two different strategies of player $i$ induce different vectors for him. If player $i$ plays $\beta T+(1-\beta) B$ (where $0<\beta<1$ ) then the induced vector for $i$ is given by $\beta f+(1-\beta) g+[\alpha(1-\beta)+\beta(1-\alpha)] \underline{M}$. Using (TI) and the construction of $f, g$ we obtain

$$
\begin{aligned}
& J_{i}(\beta f+(1-\beta) g+[\alpha(1-\beta)+\beta(1-\alpha)] \underline{M})= \\
& J_{i}(\beta f+(1-\beta) g)+[\alpha(1-\beta)+\beta(1-\alpha)] M< \\
& \beta J_{i}(f)+(1-\beta) J_{i}(g)+[\alpha(1-\beta)+\beta(1-\alpha)] M= \\
& \beta J_{i}(\alpha f+(1-\alpha)(f+\underline{M}))+(1-\beta) J_{i}(\alpha(g+\underline{M})+(1-\alpha) g \leq \\
& \max \left\{J_{i}(\alpha f+(1-\alpha)(f+\underline{M})), J_{i}(\alpha(g+\underline{M})+(1-\alpha) g)\right\} .
\end{aligned}
$$

It follows that, no matter what is the strategy of player $j$, a (strictly) best response for player $i$ is a pure strategy. But if $i$ plays a pure strategy then, by (TI), the best response for player $j$ is also a pure strategy. This implies that $G$ has no ex-ante $\mathfrak{J}$-equilibrium.

$$
(\mathbf{U A}) \Longrightarrow(\mathbf{E E})
$$

Existence of ex-ante equilibrium is guaranteed by the following argument. Since this is a standard argument, we only provide the outline of the proof and omit the details. 
For every $i \in N$, define $B R_{i}: \Sigma_{-i} \rightarrow \Sigma_{i}$ to be player $i$ 's best response correspondence. Concavity of $J_{i}$ guarantees that $B R_{i}$ is convex valued. Continuity of $J_{i}$ implies that $B R_{i}$ is upper semi-continuous and that it has compact values. (EE) is, therefore, a consequence of Brower's fixed point theorem.

\section{The proof of Theorem 2:}

\section{$(\mathbf{E E}) \Longrightarrow(\mathbf{U A})$}

Assume that (ND), (C), (H), (M) and (EE) are satisfied, and recall that each of the functionals $J_{i}$ is defined on the non-negative orthant only. (ND), (H) and (M) when combined imply that each $J_{i}$ is strictly positive on the interior of its domain.

Assume to the contrary of the theorem that there is $i \in N$ such that $J_{i}$ is not concave. We claim that it is possible to find $f, g$ as in Lemma 1 such that $f(s), g(s)>0$ for every $s \in S$. Indeed, let $f^{\prime}, g^{\prime}$ be as in the proof of Lemma 1 . Then by (C) there are $f^{\prime \prime}, g^{\prime \prime}$ such that $f^{\prime \prime}(s), g^{\prime \prime}(s)>0$ for every $s$, and such that $J_{i}\left(\alpha_{0} f^{\prime \prime}+\left(1-\alpha_{0}\right) g^{\prime \prime}\right)<$ $\alpha_{0} J_{i}\left(f^{\prime \prime}\right)+\left(1-\alpha_{0}\right) J_{i}\left(g^{\prime \prime}\right)$. Using $f^{\prime \prime}, g^{\prime \prime}$ instead of $f^{\prime}, g^{\prime}$ one obtains strictly positive vectors $f, g$ with the desired property.

We now construct a game $G$ with non-negative payoffs such that no ex-ante $\mathfrak{J}^{-}$ equilibrium exists in $G$. The information partitions, the action sets and the payoff function of player $j$ are the same as in the proof of Theorem 1. The payoff function to player $i$ is given by

$$
u_{i}\left(s, a_{i}, a_{j}\right)= \begin{cases}0 & ; a_{i}=T, a_{j}=L \\ 0 & ; a_{i}=B, a_{j}=R \\ f(s) & ; a_{i}=T, a_{j}=R \\ g(s) & ; a_{i}=B, a_{j}=L .\end{cases}
$$

Thus, the resulting bimatrix game in state $s \in S$ is given by the following diagram, where $i$ is the rows player and $j$ the columns player.

\begin{tabular}{c|c|c|}
\multicolumn{1}{c}{} & \multicolumn{1}{c}{$L$} & \multicolumn{1}{c}{$R$} \\
\cline { 2 - 3 }$T$ & 0,1 & $f(s), 0$ \\
\cline { 2 - 3 }$B$ & $g(s), 0$ & 0,1 \\
\cline { 2 - 3 } & &
\end{tabular}

Since $f(s), g(s)>0$ for every $s, J_{i}(f), J_{i}(g)>0$. This implies that there is no pure ex-ante $\mathfrak{J}$-equilibrium. $(\mathrm{H})$ and the construction of $f, g$ imply that $f, g$ are linearly independent. Thus, if player $j$ plays the strategy $\alpha L+(1-\alpha) R$ for some $\alpha \in[0,1]$, 
player's $i$ induced vector when he plays $T$ is different than the induced vector when he plays $B$.

If $i$ plays $\beta T+(1-\beta) B$ (where $0<\beta<1$ ) then the induced vector for $i$ is given by $\alpha(1-\beta) g+\beta(1-\alpha) f$. Defining $\gamma=\alpha(1-\beta)+\beta(1-\alpha)>0$ and using $(\mathrm{H})$ and the construction of $f, g$ we get

$$
\begin{aligned}
& J_{i}(\alpha(1-\beta) g+\beta(1-\alpha) f)=\gamma J_{i}\left(\frac{\alpha(1-\beta)}{\gamma} g+\frac{\beta(1-\alpha)}{\gamma} f\right)< \\
& \alpha(1-\beta) J_{i}(g)+\beta(1-\alpha) J_{i}(f)=(1-\beta) J_{i}(\alpha g)+\beta J_{i}((1-\alpha) f) \leq \\
& \max \left\{J_{i}(\alpha g), J_{i}((1-\alpha) f)\right\} .
\end{aligned}
$$

It follows that, no matter what is the strategy of player $j$, a (strictly) best response for player $i$ is a pure strategy. But if $i$ plays a pure strategy then the best response for player $j$ is also a pure strategy. This implies that $G$ has no ex-ante $\mathfrak{J}$-equilibrium.

$(\mathbf{U A}) \Longrightarrow(\mathbf{E E})$

The proof of this direction is identical to its proof in Theorem 1.

\subsection{Proofs of Section 5.}

\section{The proof of Proposition 1:}

Assume that $J_{i}$ and $J_{j}$ are maxmin functionals with respect to $P_{i}$ and $P_{j}$ respectively. If $P_{i} \neq P_{j}$ then without loss of generality there is $q \in P_{j} \backslash P_{i}$. Since $P_{i}$ is a convex compact set there exists a separating vector $f$ such that

$$
J_{i}(f)=\min _{p \in P_{i}} p \cdot f>q \cdot f \geq J_{j}(f) .
$$

Let $c$ be a constant such that $J_{i}(f)>c>J_{j}(f)$.

We shall now construct an $i j$-symmetric game $G \in \Gamma$. The information partitions of all the players are trivial. The action sets are $A_{i}=A_{j}=\{T, B\}$ and $A_{k}=\{T\}$ for every other player $k$. For an action profile $a \in A$, the payoff function of player $k(k=i, j)$ in state $s$ is given by

$$
u_{k}(s, a)= \begin{cases}c & ; a_{k}=T \\ f(s) & ; a_{k}=B .\end{cases}
$$

Thus, each of the players $i, j$ can choose between the vector $f$ and the constant vector c. The payoffs to all other players are arbitrary.

We claim that playing $T$ is a strictly dominant strategy for player $j$. Indeed, choosing $T$ gives player $j$ the payoff $c$ which is strictly higher than $J_{j}(f)$ which he gets for choosing 
B. Moreover, for every $\alpha \in(0,1)$,

$$
J_{j}(\alpha f+(1-\alpha) \underline{c})=\alpha J_{j}(f)+(1-\alpha) c<c,
$$

so player $j$ gets strictly less than $c$ if he mixes. Applying the same argument we obtain that $B$ is a strictly dominant strategy for player $i$. Thus, the unique ex-ante $\mathfrak{J}$-equilibrium of $G$ is when $j$ plays $T$ and $i$ plays $B$. This contradicts the ( $i j$-SE) axiom.

The proof of the inverse direction is standard and therefore omitted.

\section{The proof of Proposition 2:}

Given a capacity $v$, define $u_{v}(A)=\int \mathbb{1}_{A} d v$ for every $A \subseteq S$. It is shown in [28] that ${ }^{20}$ $\int f d u_{v}=\int f d v$ for every vector $f$. In particular, $u_{v}(A)=\int \mathbb{1}_{A} d v=\int \mathbb{1}_{A} d u_{v}$ for every $A \subseteq S$. Note that the normalized cover $\hat{v}=\frac{u_{v}}{u_{v}(S)}$, and that it satisfies $\hat{v}(A)=\int \mathbb{1}_{A} d \hat{v}$ for every $A \subseteq S$.

Assume that $J_{i}$ and $J_{j}$ are defined by the concave integral w.r.t. $v_{i}$ and $v_{j}$ respectively, and that $\hat{v}_{i} \neq \hat{v}_{j}$. That is, there exists $A \subset S$ such that w.l.o.g. $\hat{v}_{i}(A)>\hat{v}_{j}(A)$. Since the concave integral is piece-wise linear (Lehrer [26]), there exists $\lambda>0$ small enough such that for every $0<\lambda^{\prime} \leq \lambda$ we have that

$$
\int\left(\underline{1}+\lambda^{\prime} \mathbb{1}_{A}\right) d \hat{v}_{i}=\int \underline{1} d \hat{v}_{i}+\int \lambda^{\prime} \mathbb{1}_{A} d \hat{v}_{i}=1+\lambda^{\prime} \hat{v}_{i}(A)
$$

and

$$
\int\left(\underline{1}+\lambda^{\prime} \mathbb{1}_{A}\right) d \hat{v}_{j}=\int \underline{1} d \hat{v}_{j}+\int \lambda^{\prime} \mathbb{1}_{A} d \hat{v}_{j}=1+\lambda^{\prime} \hat{v}_{j}(A) .
$$

Thus, there exits $c>1$ such that $\int\left(\underline{1}+\lambda \mathbb{1}_{A}\right) d \hat{v}_{i}>c>\int\left(\underline{1}+\lambda \mathbb{1}_{A}\right) d \hat{v}_{j}$, which implies that

$$
J_{i}\left(\underline{1}+\lambda \mathbb{1}_{A}\right)>J_{i}(\underline{c}) \text { and } J_{j}\left(\underline{1}+\lambda \mathbb{1}_{A}\right)<J_{j}(\underline{c}) .
$$

We shall now construct an $i j$-symmetric game $G \in \Gamma$. The information partitions of all the players are trivial. The action sets are $A_{i}=A_{j}=\{T, B\}$ and $A_{k}=\{T\}$ for every other player $k$. For an action profile $a \in A$, the payoff function of player $k(k=i, j)$ in state $s$ is given by

$$
u_{k}(s, a)= \begin{cases}c & ; a_{k}=T \\ 1+\lambda \mathbb{1}_{A}(s) & ; a_{k}=B .\end{cases}
$$

Thus, players $i$ and $j$ choose between the constant vector $\underline{c}$ and $\underline{1}+\lambda \mathbb{1}_{A}$.

\footnotetext{
${ }^{20}$ The definition of the concave integral is extended naturally to set functions that need not be normalized.
} 
Let us verify that the only ex-ante $\mathfrak{J}$-equilibrium is for player $i$ to play $B$ and for player $j$ to play $T$. If player $i$ plays $\alpha B+(1-\alpha) T$ for some $0<\alpha<1$, then his induced vector is $\alpha\left(\underline{1}+\lambda \mathbb{1}_{A}\right)+(1-\alpha) \underline{c}=\alpha \underline{1}+(1-\alpha) \underline{c}+\alpha \lambda \mathbb{1}_{A}$. Since $c>1$ we get that $d=\alpha+(1-\alpha) c>1$ and $\frac{\alpha \lambda}{d}<\lambda$. This implies that

$$
J_{i}\left(\underline{1}+\frac{\alpha \lambda}{d} \mathbb{1}_{A}\right)=J_{i}(\underline{1})+J_{i}\left(\frac{\alpha \lambda}{d} \mathbb{1}_{A}\right) .
$$

Now,

$$
\begin{gathered}
J_{i}\left(\alpha\left(\underline{1}+\lambda \mathbb{1}_{A}\right)+(1-\alpha) \underline{c}\right)=J_{i}\left(\alpha \underline{1}+(1-\alpha) \underline{c}+\alpha \lambda \mathbb{1}_{A}\right)=J_{i}\left(\underline{d}+\alpha \lambda \mathbb{1}_{A}\right)= \\
d J_{i}\left(\underline{1}+\frac{\alpha \lambda}{d} \mathbb{1}_{A}\right)=d J_{i}(\underline{1})+J_{i}\left(\alpha \lambda \mathbb{1}_{A}\right)=\alpha+(1-\alpha) c+\alpha \lambda \hat{v}_{i}(A)= \\
\alpha J_{i}\left(\underline{1}+\lambda \mathbb{1}_{A}\right)+(1-\alpha) J_{i}(\underline{c}) .
\end{gathered}
$$

In particular, since $J_{i}\left(\underline{1}+\lambda \mathbb{1}_{A}\right)>J_{i}(\underline{c})$ player $i$ would prefer $\underline{1}+\lambda \mathbb{1}_{A}$ to any convex combination with $\underline{c}$. This means that $B$ is a strictly dominant strategy for $i$. In the same way it can be shown that $T$ is a strictly dominant strategy for $j$.

The proof of the inverse direction is standard and therefore omitted.

\subsection{The proof of Proposition 3.}

The proof is based on the main proposition in Samet [36]. Applying Samet's result, we know that $\bigcap_{i \in N} P_{i}=\emptyset$ iff there are $\left\{f_{i}\right\}_{i \in N} \subseteq \mathbb{R}^{m}$ such that $\sum_{i \in N} f_{i}=0$ and $p_{i} \cdot f_{i}>0$ for every $p_{i} \in P_{i}$ and for $i=1, \ldots, n$.

Assume $\bigcap_{i \in N} P_{i}=\emptyset$. Define $G$ to be the game where each player has only one action, and where player $i$ 's payoff in state $s$ is $f_{i}(s)$. Obviously, $G$ is a zero-sum game and in the (unique) strategy profile $\sigma$ we have $J_{i}\left(f_{i}^{(\sigma)}\right)>0$ for every $i \in N$.

Conversely, assume $\bigcap_{i \in N} P_{i} \neq \emptyset$. Then for any profile $\sigma$ in some zero-sum game $G$ we have that $\sum_{i \in N} f_{i}^{(\sigma)}=0$. By Samet's proposition, it can't be that $J_{i}\left(f_{i}^{(\sigma)}\right)>0$ for all $i \in N$.

\section{REFERENCES}

[1] P. Artzner, F. Delbaen, J.M. Eber, D. Heath, Coherent measures of risk, Mathematical Finance 9 (1999), 203-228.

[2] R. J. Aumann, Correlated equilibrium as an expression of Bayesian rationality, Econometrica 55 (1987), 1-18. 
[3] Y. Azrieli, E. Lehrer, Extendable cooperative games, Journal of Public Economic Theory 9 (2007), 1069-1078.

[4] S. Bade, Ambiguous act equilibria, (2008) Manuscript.

[5] S. Bade, Electoral competition with uncertainty averse parties, (2008) Manuscript.

[6] A. Billot, A. Chateauneuf, I. Gilboa, J.M. Tallon, Sharing beliefs: Between agreeing and disagreeing, Econometrica 68 (2000), 685-694.

[7] S. Bose, E. Ozdenoren, A. Pape, Optimal auctions with ambiguity, Theoretical Economics 1 (2006), 411-438.

[8] S. Cerreia, F. Maccheroni, M. Marinacci, L. Montrucchio, Uncertainty averse preferences, (2008) Manuscript.

[9] F. Delbaen, Coherent risk measures on general probability spaces, in: K. Sandmann et al. (Eds.), Advances in Finance and Stochastics (Essays in Honour of Dieter Sondermann), Springer, pp1-37.

[10] A. Dempster, Upper and lower probabilities induced by multi-valued mapping, Annals of Mathematical Statistics 38 (1967), 325-339.

[11] D. Ellsberg, Risk, ambiguity, and the Savage axioms, Quarterly Journal of Economics 75 (1961), 643-669.

[12] L.G. Epstein, A definition of uncertainty aversion, Review of Economic Stusied 66 (1999), 579-608.

[13] L.G. Epstein, M. Le Breton Dynamically consistent beliefs must be bayesian, Journal of Economic Theory 61 (1993), 1-22.

[14] L.G. Epstein, T. Wang, Beliefs about beliefs without probabilities, Econometrica 64 (1996), 13431373.

[15] R. Fagin, J.Y. Halpern, A new approach to updating beliefs, in: P.P. Bonissone, M. Henrion, L.N. Kanal, J.F. Lemmer (Eds.), Proc. 6th conference uncertainty and AI, 1991, pp347-374.

[16] H. Föllmer, A. Scheid, Convex measures of risk and trading constraints, Finance and Stochastics 6 (2002), 429-447.

[17] P. Ghirardato, M. Marinacci, Ambiguity made precise: A comparative foundation, Journal of Economic Theory 102 (2002), 251-289.

[18] I. Gilboa, D. Schmeidler, Maxmin expected utility with a non-unique prior, Journal of Mathematical Economics 18 (1989), 141-153.

[19] I. Gilboa, D. Schmeidler, Updating ambiguous beliefs, Journal of Economic Theory 59 (1993), 33-49.

[20] E. Hanany, P. Klibanoff, Updating ambiguity averse preferences, (2007) Manuscript.

[21] J.C. Harsanyi, Games with incomplete information played by "Bayesian" players I-III, Management Science 14 (1967), 159-182, 320-334, 486-502.

[22] A. Kajii, T. Ui, Incomplete information games with multiple priors, Japanese Economic Review 56 (2005), 332-351.

[23] A. Kajii, T. Ui, Agreeable bets with multiple priors, Journal of Economic Theory 128 (2006), 299-305. 
[24] A. Kajii, T. Ui, Interim efficient allocations under uncertainty, Journal of Economic Theory, (2008) Forthcoming.

[25] E. Karni, State-dependent utility, in: P. Anand, P. K. Pattanaik, C. Puppe (Eds.), Handbook of rational and social choice, Oxford University Press, Oxford, Forthcoming.

[26] E. Lehrer, A new integral for capacities, Economic Theory, (2007) Forthcoming.

[27] E. Lehrer, Partially-specified probabilities: Decisions and games, (2006) Manuscript.

[28] E. Lehrer, R. Teper, The concave integral over large spaces, Fuzzy Sets and Systems 159 (2008), 2130-2144.

[29] K.C. Lo, Sealed bid auctions with uncertainty averse bidders, Economic Theory 12 (1998), 1-20.

[30] F. Maccheroni, M. Marinacci, A. Rustichini, Ambiguity aversion, robustness, and the variational representation of preferences, Econometrica 74 (2006), 1447-1498.

[31] P.R. Milgrom, R.J. Weber, Distributional strategies in games with incomplete information, Mathematics of Operations Research 10 (1985), 619-632.

[32] S. Mukerji, J. M. Tallon, An overview of economic applications of David Schmeidlers models of decision making under uncertainty, in: I. Gilboa (Editor), Uncertainty in Economic Theory: A collection of essays in honor of David Schmeidlers 65 th birthday, Routledge Publishers, London, 2004, pp283-302.

[33] T. Rader, Theory of Microeconomics, Academic Press, New York, 1972.

[34] L. Rigotti, C. Shannon, T. Strzalecki, Subjective beliefs and ex-ante trade, Econometrica, (2008) Forthcoming.

[35] A. Salo, M. Weber, Ambiguity aversion in first-price sealed-bid auctions, Journal of Risk and Uncertainty 11 (1995), 123-137.

[36] D. Samet, Common priors and seperation of convex sets, Games and Economic Behavior 24 (1998), 172-174.

[37] R. Sarin, P. Wakker, Dynamic choice and nonexpected utility, Journal of Risk and Uncertainty 17 (1998), 87-119.

[38] D. Schmeidler, Subjective probability and expected utility without additivity, Econometrica 57 (1989), 571-587.

[39] G. Shafer, A Mathematical Theory of Evidence, Princeton University Press, Princeton, NJ., 1976.

[40] W.W. Sharkey, Cooperative games with large cores, International Journal of Game Theory 11 (1982), 175-182.

[41] W.R. Shephard, Theory of Cost and Production Functions, Princton University Press, Princeton, NJ., 1970.

[42] T. Wang, Conditional preferences and updating, Journal of Economic Theory 108 (2003), 286-321. 